\title{
Identification of the Transverse Distributed Load in the Euler-Bernoulli Beam Equation from Boundary Measurement
}

\author{
Yeşim Saraç and S. Şule Şener
}

\begin{abstract}
This paper is concerned with an optimal control problem for the Euler-Bernoulli beam equation. We assume that the transverse distributed load is a control function. We prove the existence of the unique optimal solution in the suitable set of admissible control. We get the gradient of the cost functional by using the adjoint problem.
\end{abstract}

Index Terms-Euler-Bernoulli beam equation, optimization, boundary measurement, frechet differentiability.

\section{INTRODUCTION AND OPTIMAL CONTROL PROBLEM FORMULATION}

The optimal control problems for the ordinary differential equations (ODEs) have been discussed in many papers. Yousefi et al. [1] studied the optimal control problem for the system of the first-order linear differential equations. The control function is at the right hand side of the system. They have tested presented results for the first-order differential equation and the system of the linear differential equations in the numerical examples. The problems of finding the leading coefficients in second-order ODEs are investigated in [2-4]. In [5], Papanicolau solved the problem of determining of the flexural rigidity coefficient $a(x)$ in the equation $\left(a(x) u^{\prime \prime}(x)\right)^{\prime \prime}=\lambda p(x) u(x)$, where $u(x)$ is the deflection of the beam. Inverse coefficient identification problems governed by the steady state Euler-Bernoulli beam equation are studied in [6-10].

We consider the following optimal control problem of minimizing a quadratic cost functional

$$
J_{\alpha}(f)=\left[u(b ; f)-u_{b}\right]^{2}+\alpha \int_{a}^{b} f^{2}(x) d x
$$

Subject to a linear forth-order ODE system

$$
\begin{aligned}
& \left(a(x) u^{\prime \prime}\right)^{\prime \prime}+q(x) u=f(x), \quad x \in[a, b] \\
& u(a)=0,\left.\quad\left(a(x) u^{\prime \prime}\right)\right|_{x=a}=0, \\
& u^{\prime}(b)=0,\left.\left(a(x) u^{\prime \prime}\right)^{\prime}\right|_{x=b}=0
\end{aligned}
$$

where $q$ is the given function and $u_{b}$ is the given constant. In the system (2), the functions $a, q$ and $f$ represent the flexural rigidity, foundation modulus and the transversely distributed load for vibrating beam, respectively.

Manuscript received November 15, 2017; revised January 12, 2018.

Atatürk University is with Faculty of Science, Department of Mathematics, 25240, Erzurum, Turkey (e-mail: ysarac@ atauni.edu.tr).
$L_{2}[a, b]$ is the Banach space consinsting of all measurable functions on $[a, b]$ having the inner product and the norm, respectively, by the following equalities

$$
\begin{aligned}
\langle u, v\rangle_{L_{2}[a, b]} & =\int_{a}^{b} u(x) v(x) d x \\
\|u\|_{L_{2}[a, b]} & =\sqrt{\langle u, u\rangle_{L_{2}[a, b]}}
\end{aligned}
$$

for $u, v \in L_{2}[a, b]$.

We focus on finding the transversely distributed load $f(x)$ of a beam from the admissible functions set

$$
F=\left\{f: f(x) \in L_{2}[a, b],\|f\|_{L_{2}[a, b]} \leq k\right\}
$$

where $k$ is a constant. The scalar product and the norm in the set $F$ will be respectively defined as follows

$$
\left\langle f_{1}, f_{2}\right\rangle_{F}=\int_{a}^{b} f_{1}(x) f_{2}(x) d x
$$

and

$$
\|f\|_{L_{2}[a, b]}=\sqrt{\langle f, f\rangle_{F}}
$$

for $f_{1}, f_{2} \in F$.

Let $u(x ; f)$ denote the solution of the boundary value problem (2) at the point $x$ corresponding to a given control $f \in F$. In (1), the number $\alpha>0$ is the parameter of regularization and it can be found by the Tikhonov regularization method [11]. This number provides a fair balance between minimizing the norm $\|f\|_{L_{2}[a, b]}^{2}$ and minimizing the residual $\left[u(b ; f)-u_{b}\right]^{2}$.

It is assumed that $f, q, a \in L_{2}[a, b]$ and the functions $a(x)$ and $q(x)$ satisfies the conditions

$$
0<a_{0} \leq a(x) \leq a_{1}
$$

and

$$
0<q_{0} \leq q(x) \leq q_{1}
$$

for $x \in[a, b]$, where $a_{0}, a_{1}, q_{0}$ and $q_{1}$ are some constants. Then the problem (2) has a unique weak solution $u \in$ $\widetilde{H}^{2}[a, b]$ and this solution satisfies the following integral identity 


$$
\begin{gathered}
\int_{a}^{b}\left[a(x) u^{\prime \prime}(x) v^{\prime \prime}(x)+q(x) u(x) v(x)\right] d x \\
=\int_{a}^{b} f(x) v(x) d x, \quad \forall v \in \widetilde{H}^{2}[a, b]
\end{gathered}
$$

where

$$
\widetilde{H}^{2}[a, b]:=\left\{u \in H^{2}[a, b]: u(a)=u^{\prime}(b)=0\right\} .
$$

The plan of the paper is as follows: In section 2, we prove that the cost functional $J_{\alpha}(f)$ is the continuous and strongly convex and thus the uniqueness of the optimal solution. In section 3 , we find the gradient of the cost functional via adjoint problem approach and constitute the minimizing sequence for the functional (1).

\section{WELL-POSEDNESS OF THE OPTIMAL CONTROL PROBLEM}

The aim of this section is to prove the existence, uniqueness and stability of the solution of the optimal control problem (1)-(2). For this purpose, we show that the cost functional $J_{\alpha}(f)$ is continuous and strongly convex.

Let's give the increment $\Delta f$ to $f$ such that $f+\Delta f \in F$ show the solution of (2) corresponding $f+\Delta f$ by $u_{\Delta}=u(x ; f+\Delta f)$. Then the function $\Delta u=u_{\Delta}-u$ will be the solution of the following difference problem:

$$
\begin{aligned}
& \left(a(x) \Delta u^{\prime \prime}\right)^{\prime \prime}+q(x) \Delta u=\Delta f(x), x \in[a, b] \\
& \Delta u(a)=0,\left.\left(a(x) \Delta u^{\prime \prime}\right)\right|_{x=a}=0, \\
& \Delta u^{\prime}(b)=0,\left.\left(a(x) \Delta u^{\prime \prime}\right)^{\prime}\right|_{x=b}=0
\end{aligned}
$$

Lemma 2.1. Let $\Delta u=\Delta u(x ; f)$ be the solution of the problem (6) corresponding to a given $f \in F$. Then the following estimate is valid:

$$
[\Delta u(b ; f)]^{2} \leq c_{1}\|\Delta f\|_{L_{2}[a, b]}^{2}
$$

where $c_{1}=\frac{\varepsilon_{1}(b-a)^{3}}{2 a_{0}}$.

Proof: Let us multiply both sides of the difference problem (6) by $\Delta u$ and integrate on $[a, b]$; we can easily write that

$$
\begin{gathered}
\int_{a}^{b} a(x)\left(\Delta u^{\prime \prime}\right)^{2} d x+\int_{a}^{b} q(x)(\Delta u)^{2} d x \\
=\int_{a}^{b} \Delta f(x) \Delta u d x .
\end{gathered}
$$

Let us apply the Cauchy-Schwarz inequality to the righthand side and use conditions (4) and (5), then we have

$$
\begin{gathered}
a_{0}\left\|\Delta u^{\prime \prime}\right\|_{L_{2}[a, b]}^{2}+q_{0}\|\Delta u\|_{L_{2}[a, b]}^{2} \\
\leq\|\Delta f\|_{L_{2}[a, b]}\|\Delta u\|_{L_{2}[a, b]} .
\end{gathered}
$$

Appling the Cauchy inequality to the right-hand side, we get

$$
\begin{aligned}
& a_{0}\left\|\Delta u^{\prime \prime}\right\|_{L_{2}[a, b]}^{2}+q_{0}\|\Delta u\|_{L_{2}[a, b]}^{2} \\
& \quad \leq \frac{\varepsilon_{1}}{2}\|\Delta f\|_{L_{2}[a, b]}^{2}+\frac{1}{2 \varepsilon_{1}}\|\Delta u\|_{L_{2}[a, b]}^{2}
\end{aligned}
$$

We can easily write

$$
\left\|\Delta u^{\prime \prime}\right\|_{L_{2}[a, b]}^{2} \leq \frac{\varepsilon_{1}}{2 a_{0}}\|\Delta f\|_{L_{2}[a, b]}^{2}
$$

where $\varepsilon_{1} \geq \frac{1}{2 q_{0}}$. From the inequality $\left\|\Delta u^{\prime}\right\|_{L_{2}[a, b]}^{2} \leq$ $(b-a)^{2}\left\|\Delta u^{\prime \prime}\right\|_{L_{2}[a, b]}^{2}$, we have

$$
\begin{aligned}
{[\Delta u(b ; f)]^{2} } & =\left[\int_{a}^{b} \Delta u^{\prime} d x\right]^{2} \\
& \leq(b-a)\left\|\Delta u^{\prime}\right\|_{L_{2}[a, b]}^{2} \\
& \leq(b-a)^{3}\left\|\Delta u^{\prime \prime}\right\|_{L_{2}[a, b]}^{2}
\end{aligned}
$$

The proof follow from the inequality (8).

Lemma 2.2. The difference $\Delta J_{\alpha}(f)=J_{\alpha}(f+\Delta f)-$ $J_{\alpha}(f)$ of the cost functional $J_{\alpha}(f)$ satisfies the following inequality;

$$
\Delta J_{\alpha}(f) \leq c_{2}\|\Delta f\|_{L_{2}[a, b]}+c_{3}\|\Delta f\|_{L_{2}[a, b]}^{2}
$$

where $c_{2}$ and $c_{3}$ are constants independent of $\Delta f$.

Proof. It is easily seen that

$$
\begin{aligned}
\Delta J_{\alpha}(f) & =\left[u(b ; f+\Delta f)-u_{b}\right]^{2}-\left[u(b ; f)-u_{b}\right]^{2} \\
& +\alpha\left(\|f+\Delta f\|_{L_{2}[a, b]}^{2}-\|f\|_{L_{2}[a, b]}^{2}\right) .
\end{aligned}
$$

We can write

$$
\begin{aligned}
\Delta J_{\alpha}(f) & =\left[2 u(b ; f)-2 u_{b}+\Delta u(b ; f)\right] \Delta u(b ; f) \\
& +\alpha\left(\langle 2 f, \Delta f\rangle_{F}+\|\Delta f\|_{L_{2}[a, b]}^{2}\right) .
\end{aligned}
$$

Using (3) and (7), we can write

$$
\begin{aligned}
\left|\Delta J_{\alpha}(f)\right| & \leq\left(2 m \sqrt{c_{1}}+2 \alpha k\right)\|\Delta f\|_{L_{2}[a, b]} \\
& +\left(c_{1}+\alpha\right)\|\Delta f\|_{L_{2}[a, b]}^{2}
\end{aligned}
$$

For $m=|u(b ; f)|+\left|u_{b}\right|$. If taking as $c_{2}=2 m \sqrt{c_{1}}+$ $2 \alpha k$ and $c_{3}=c_{1}+\alpha$ the estimate (9) is obtained.

The inequality (9) implies the continuity of the functional (1).

Lemma 2.3: The functional $J_{\alpha}(f)$ is strongly convex on F.

Proof: We know that the set $F$ is convex [12]. For all $f_{1}, f_{2} \in F$ and $\lambda \in[0,1]$, we can write 


$$
\begin{aligned}
J_{\alpha} & \left(\lambda f_{1}+(1-\lambda) f_{2}\right) \\
= & {\left[u\left(b ; \lambda f_{1}+(1-\lambda) f_{2}\right)-u_{b}\right]^{2} } \\
& +\alpha\left\|\lambda f_{1}+(1-\lambda) f_{2}\right\|_{L_{2}[a, b]}^{2} \\
= & \lambda^{2}\left[u\left(b ; f_{1}\right)-u_{b}\right]^{2}+(1-\lambda)^{2}\left[u\left(b ; f_{2}\right)-u_{b}\right]^{2} \\
& +2 \lambda(1-\lambda)\left[u\left(b ; f_{1}\right)-u_{b}\right]\left[u\left(b ; f_{2}\right)-u_{b}\right] \\
& +\alpha\left(\lambda\left\|f_{1}\right\|_{L_{2}[a, b]}^{2}+(1-\lambda)\left\|f_{2}\right\|_{L_{2}[a, b]}{ }^{2}\right) \\
& -\alpha \lambda(1-\lambda)\left\|f_{1}-f_{2}\right\|_{L_{2}[a, b]}^{2} \\
\leq & \lambda\left(\left[u\left(b ; f_{1}\right)-u_{b}\right]^{2}+\alpha\left\|_{1}\right\|_{L_{2}[a, b]}\right) \\
& +(1-\lambda)\left(\left[u\left(b ; f_{2}\right)-u_{b}\right]^{2}+\alpha f_{2} \|_{L_{2}[a, b]}^{2}\right) \\
& -\alpha \lambda(1-\lambda)\left\|f_{1}-f_{2}\right\|_{L_{2}[a, b]}^{2}
\end{aligned}
$$

So we have

$$
\begin{aligned}
J_{\alpha}\left(\lambda f_{1}+(1-\lambda) f_{2}\right) & \leq \lambda J_{\alpha}\left(f_{1}\right)+(1-\lambda) J_{\alpha}\left(f_{2}\right) \\
& -\alpha \lambda(1-\lambda)\left\|f_{1}-f_{2}\right\|_{L_{2}[a, b]}^{2}
\end{aligned}
$$

For all $f_{1}, f_{2} \in F$ and $\lambda \in[0,1]$. This implies the strongly convexity of the cost functional $J_{\alpha}(f)$ for the constant $\chi=\alpha$.

So the conditions of the following theorem [13] hold and this show that the optimal control problem (1)-(2) is wellposed, namely existence, uniqueness and stability of the optimal solution.

Theorem 2.4: Suppose that $F \subseteq H$ is a closed, convex set in a Hilbert space $H$ and $J_{\alpha}(f)$ is a continuous and strongly convex functional with constant $\chi>0$ on this set. Then

a) The functional $J_{\alpha}(f)$ is bounded below on $F$

$$
\inf _{F} J_{\alpha}(f)=J_{\alpha}^{*}>-\infty .
$$

b) For a unique $f^{*} \in F, J_{\alpha}\left(f^{*}\right)=J_{\alpha}{ }^{*}$.

c) Any minimizing sequence $\left\{f_{k}\right\}$ converges strongly to the element $f^{*}$ in $H$ and the following estimate is valid;

$$
\left\|f_{k}-f^{*}\right\|_{L_{2}[a, b]}^{2} \leq \frac{2}{\chi}\left[J_{\alpha}\left(f_{k}\right)-J_{\alpha}\left(f^{*}\right)\right], \quad k=1,2, \ldots
$$

\section{FRECHET DIFFERENTIABILITY OF THE COST FUNCTIONAL}

In this section, we get the Frechet differential of the cost functional by using the adjoint problem approach and constitute the minimizing sequence which converges the optimal solution.

Considering the definition of the Frechet differential, we need to transform the right-hand side of the (10) into the following form:

$$
\Delta J_{\alpha}(f)=\left\langle J_{\alpha}^{\prime}(f), \Delta f\right\rangle_{F}+o\left(\|\Delta f\|_{F}^{2}\right) .
$$

Lemma 3.1. Let $f, f+\Delta f \in F$ be given elements. If $u=u(x ; f)$ is the corresponding solution of the problem (2) and $\eta=\eta(x ; f)$ is the solution of the adjoint problem

$$
\begin{aligned}
& \left(a(x) \eta^{\prime \prime}\right)^{\prime \prime}+q(x) \eta=0, \quad x \in[a, b] \\
& \eta(a)=0,\left.\quad\left(a(x) \eta^{\prime \prime}\right)\right|_{x=a}=0, \eta^{\prime}(b)=0, \\
& \left.\left(a(x) \eta^{\prime \prime}\right)^{\prime}\right|_{x=b}=2 u(b ; f)-2 u_{b}
\end{aligned}
$$

then for all $f \in F$ the following equality valids:

$$
\left[2 u(b ; f)-2 u_{b}\right] \Delta u(b ; f)=\int_{a}^{b}-\eta \Delta f d x .
$$

Proof. From the boundary condition of the problem (11), we write

$$
\begin{aligned}
& {\left[2 u(b ; f)-2 u_{b}\right] \Delta u(b ; f)} \\
& =\int_{a}^{b} \frac{d}{d x}\left[\left(a(x) \eta^{\prime \prime}\right)^{\prime} \Delta u\right] d x \\
& =\int_{a}^{b}\left[\left(a(x) \eta^{\prime \prime}\right)^{\prime \prime} \Delta u+\left(a(x) \eta^{\prime \prime}\right)^{\prime} \Delta u^{\prime}\right] d x .
\end{aligned}
$$

Let us use the difference problem (6) and adjoint problem (11), we get

$$
\begin{aligned}
& {\left[2 u(b ; f)-2 u_{b}\right] \Delta u(b ; f)} \\
& \quad=\int_{a}^{b}\left[-q(x) \Delta u \eta-\left(a(x) \Delta u^{\prime \prime}\right)^{\prime \prime} \eta\right] d x \\
& \quad=\int_{a}^{b}-\eta \Delta f d x
\end{aligned}
$$

The proof is done.

Now we use the equality (12) on the right-hand side of formula (10) to obtain the first variation of the cost functional $J_{\alpha}(f)$. Then we have

$$
\begin{aligned}
\Delta J_{\alpha}(f) & =\int_{a}^{b}-\eta \Delta f d x++[\Delta u(b ; f)]^{2} \\
& +\alpha\left(\langle 2 f, \Delta f\rangle_{F}+\|\Delta f\|_{F}^{2}\right)
\end{aligned}
$$

Taking into account the definition of the scalar product in $F$, we write

$$
\begin{aligned}
\Delta J_{\alpha}(f)= & \langle-\eta+2 \alpha f, \Delta f\rangle_{F}+[\Delta u(b ; f)]^{2} \\
& +\alpha\|\Delta f\|^{2}{ }_{F}
\end{aligned}
$$

The Lemma 2.1 implies that the second term in (13) is bounded by term $o\left(\|\Delta f\|_{F}^{2}\right)$. So Frechet differential at $f \in F$ of the cost functional $J_{\alpha}(f)$ can be defined as follows:

$$
J_{\alpha}^{\prime}(f)=-\eta+2 \alpha f .
$$

Now we set a minimizing sequence for minimization problem (1)-(2) according to the gradient method by 


$$
f_{k+1}=f_{k}-\beta_{k} J_{\alpha}^{\prime}\left(f_{k}\right), k=0,1,2, \cdots
$$

where $f_{0} \in F$ is a given initial iteration and the constant $\beta_{k}$ be found from $J_{\alpha}\left(f_{k+1}\right)<J_{\alpha}\left(f_{k}\right)$ condition. Convergence of this sequence has been given in the Theorem 2.4. Concerning the choice of the parameter $\beta_{k}$, there are several possibilities and these can be found in any optimization books.

\section{CONCLUSION}

In Euler-Bernoulli beam equation, the transverse distributed load can be controlled from the boundary measurement by using the chosen cost functional (1). The regularization parameter $\alpha$ is the strong convexity constant for the cost functional (1). According to Theorem 2.4 the minimizing sequence in (14) converges the optimal solution.

\section{REFERENCES}

[1] S. A. Yousefi, M. Dehghan, and A. Lofti, "Finding the optimal control of linear systems via he's variational iteration method," International Journal of Computer Mathematics, vol. 87, no. 5, pp. 1042-1050, 2010.

[2] A. Hasanov and T. S. Shores, "Solution of an inverse coefficient problem for an ordinary differential equation," Applicable Analysis, vol. 67, pp. 11-20, 1997.

[3] Z. Seyidmamedov and A. Hasanov, "Determination of leading coefficients in sturm-liouville operator from boundary measurements, I. A stripping algorithm," Applied Mathematics and Computation, vol 125 , pp.1-21, 2002

[4] A. Hasanov and Z. Seyidmamedov, "Determination of leading coefficients in sturm-liouville operator from boundary measurements, II. Unicity and an engineering approach," Applied Mathematics and Computation, vol. 125, pp. 23-34, 2002.

[5] V. Papanicolau, "The spectral theory of the vibrating periodic beam," Communications in Mathematical Physics, vol. 170, pp. 359-373, 1995.
[6] D. Lesnic, "Determination of the flexural rigidity of a beam from limited boundary measurements," J. Appl. Math. And Computing, vol. 20, pp. 17-34, 2006.

[7] D. Lesnic and A. Hasanov, "Determination of the leading coefficient in fourth-order Sturm-Liouville operator from boundary measurements," Inverse Problems in Science Engineering, vol. 16, no. 4, pp. 413-424, 2008.

[8] D. Lesnic, L. Elliott, and D. B. Ingham, "Analysis of coefficient identification problems associated to the inverse Euler-Bernoulli beam theory," IMA Journal of Applied Mathematics, vol. 62, pp. 101116,1999

[9] T. Marinov and A. S. Vatsala, "Inverse problem for coefficient identification in the euler-bernoulli equation," An International Journal Computers and Mathematics with Applications, vol. 56, pp. 400-410, 2008.

[10] T. T. Marinov and R. Marinova, "Coefficient identification in EulerBernoulli equation from over-posed data," Journal of Computational and Applied Mathematics, vol. 235, pp. 450-459, 2008.

[11] A. N. Tikhonov and A. A. Samarskii, Equations of Mathematical Physics, Dover Publications, New York, 1963.

[12] K. Yosida, Functional Analysis, Springer-Verlag, New York, 1980.

[13] F.P. Vasilyev, Methods for Solution of Extremal Problems, Nauka, Moskova,1980.

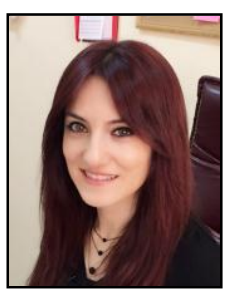

Yeşim Saraç was born in Gümüşhane, Turkey. She received the $\mathrm{Ph} . \mathrm{D}$. degree in applied mathematics from Atatürk University.

She is an assistant professor in the Department of Mathematics of Atatürk University. She is interested in optimal control, partial differential equations, numerical analysis.

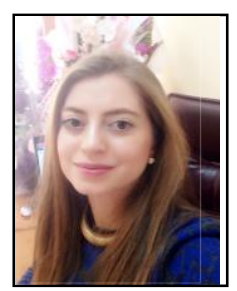

S. Şule Şener was born in Erzurum, Turkey. She received the Ph.D. degree in applied mathematics from Atatürk University.

She is an assistant professor in the Department of Mathematics of Atatürk University. She is interested in partial differential equations, optimization, mathematical modelling. 